\title{
Discussion on Optical Fiber Transmission Network Technology for 5G Mobile Communication Fronthaul
}

\author{
Lin Yuan", Xu Jun², Cheng Shuaipeng ${ }^{3}$, Xu Changbin ${ }^{4}$ \\ ${ }^{1}$ Jiangxi Teachers College, Jiangxi Yingtan 335000, China \\ ${ }^{2}$ Jiangxi Ruiyuan Precision Machining Co.,Ltd. \\ ${ }^{3}$ Jiangxi Teachers College, Jiangxi Yingtan 335000, China \\ ${ }^{4}$ Jiangxi Teachers College, Jiangxi Yingtan 335000, China)
}

Keywords: 5G mobile communication; fiber optic transmission network; key technologies

\begin{abstract}
The construction of network information technology is continuously progressing. The application scope of $5 \mathrm{G}$ is gradually being promoted. Compared with $3 \mathrm{G}$ and $4 \mathrm{G}$ technologies, the core of $5 \mathrm{G}$ technology forms is mobile communication fronthaul technology forms under fiber optic transmission. This technology form has an important role and is supported by information technology. Promote the rational application of the overall technology In this study, the key technology of $5 \mathrm{G}$ mobile communication fronthaul is used as the basis to analyze how to realize the rational application of technology.
\end{abstract}

\subsection{G mobile communication overview}

5G technology is mainly used in the Internet of Things, real-time connection, user experience, ultra-high-speed communication, and practicality of large-scale crowds. According to relevant data research, in order to achieve 5G communication technology, we must first meet the following basic requirements: First, the communication transmission speed must reach $10 \mathrm{~GB} / \mathrm{s}$, the spectrum efficiency must be increased to about 10 times, and the energy efficiency also needs to be improved 10 times, network capacity needs to be increased by 1000 times, and latency needs to be less than 5 milliseconds.[1] It can be seen that compared to 4G mobile communications, 5G does not just mean an increase in Internet speed, but also means a better user experience and more network applications. In order to effectively reduce the resources and application configuration of 5G networks and continuously improve their intelligence, researchers have already identified some specific trends.

According to the relevant information, it is not difficult to find out that the future development trend of 5G networks can be found. It mainly includes the following aspects: First, the continuous development of wireless access technology, non-orthogonal multiple access technology may be used in 5G networks, which is of great significance to the improvement of resource utilization efficiency. [2]Second, the coverage of the cellular network will continue to shrink. Due to the continuous advancement of small cell technology, it is a general trend to reduce the coverage of the cellular network in future network technologies, especially in some regions with a relatively high degree of commercialization. Small cell networks often exhibit dense or ultra-dense distribution characteristics, so 5G mobile communications will inevitably adopt this model. [3]Third, in order to improve resource utilization efficiency and user requirements for data transmission speed, 5G mobile communication will inevitably use a combination of cellular and different technical access methods, so-called heterogeneity. Fourth, intelligent development. In order to ensure energy savings in heterogeneous networks and ensure user experience, 5G communication networks will introduce intelligence into the architecture.

\section{$25 G$ network architecture}

At present, scholars at home and abroad have a consistent understanding of 5G network architecture, which is obtained by adding new technologies through the evolution of existing RATs. Although worldwide research on 
$5 \mathrm{G}$ is still in its infancy, some results have been achieved. For example, researchers at the current stage have proposed a new heterogeneous LTE-B network architecture. Based on the conclusions drawn from relevant research on 5G at this stage, the author has studied the basic architecture of 5G networks. [4]The author believes that in the future 5G network, it will be divided into three different modules: network deployment scenarios, access networks, and core networks.

\section{1 network deployment scenarios}

In the deployment of network scenarios, we need to distinguish between indoor scenarios and outdoor scenarios. During the deployment of indoor scenarios, users can communicate through the APP installed in the outdoor antenna. In this way, the advantages of short-range communication can be fully utilized to achieve high-speed transmission of information.

\subsection{Access network design}

In the process of designing the access network, you need to pay attention to several characteristics: First, the integration of multiple access technologies: mainly including 2G, 3G, 4G, and wife and other access methods, and then in the process of access Can be accessed using a single wireless controller. Second, the allocation of virtual resources through base stations: On the basis of deploying centralized base stations, SDR will gradually realize the processing of baseband and gradually transition to virtualized base stations with real-time cloud architecture. Third, content edge caching and delivery. Content storage and distribution functions in traditional mobile networks will be transferred to the access network, and information will be pushed according to the actual needs of users, thereby continuously improving the user experience.

\subsection{Core Network Design}

In 5G mobile networks, the change of the core network is mainly driven by NFV and SDN, which mainly includes several different characteristics: Second, the development of control and forwarding is separate. There is a comparative between SDN and the traditional network system.[5] The big difference is that the control and forwarding are integrated in the traditional network system, but in SDN, the centralized network controller can realize the distribution of data traffic after the network is separated, thereby achieving various functions.

\section{Different types of key technologies for network fronthaul}

\subsection{Centralized Basic Architecture}

The composition of traditional base stations is divided into different parts. In the basic design, the design of the baseband unit is performed. In digital signal processing, you need to understand the composition of the information system. Taking the RRU system as an example, it is accepted in the system radio frequency transmission. Functional analysis and traditional CPRI connection protocol can promote the consistency of interface design. In the process of network development, network providers are required to adopt the CPRI connection protocol to ensure the rationality of the basic framework.[6]

The centralized management method is a new radio access network design form in the current network environment. In the design, C-RAN uses a centralized operation form, a collaborative operation form, etc. for calculation. In the current access network design, it is required to reduce the number of base station equipment rooms. In the design of energy consumption, through the writing and virtualization technology, resource sharing and reasonable allocation can be ensured. In the networked operation management C-RAN design form, which meets the requirements of the mobile Internet design, in the current rapid development process, there may be multiple pressures, so the overall sustainable management has benefit value analysis, which can meet specific process requirements. 


\subsection{Constructing the Pure Digital Signal CPRI Protocol Fronthaul Technology}

\subsubsection{WDM-based fronthaul technology}

In the process of short-term benefit analysis, the network design form needs to be reasonably preset. In the process of current information system analysis, in the process of basic method construction, it is necessary to pay attention to the effective application of the structural method. [7]Need to adapt to outdoor installation requirements. In the system application, there are two forms of links, and the demand for optical fibers is controlled in the design of the scheme. Only by doing a good job of network maintenance can the feasibility be improved. SG mobile communication does not have many mid-sites, and the types and number of optical modules involved in it increase. It is difficult to meet the transmission requirements only through wavelength division multiplexing. What needs to be performed in the actual presetting process is a reasonable scheme multiplexing technology. In applications, if the wavelength is not determined, it needs to be reasonably distinguished.

\subsubsection{Forwarding Technology Based on Time Division Multiplexing}

The form of fronthaul technology based on time division multiplexing meets specific requirements. In the application of multiplexing technology, we develop comprehensive countermeasures based on TDM, combining the requirements of Ethernet and TDM technology, etc. After technical innovation, we can determine reasonable solutions. During the process of building the system framework, we can fully respond The existing mobile communication system is preset, and finally the converter is converted and sent to the RRH.

\subsection{Optical carrier IF for pure analog signal transmission fronthaul technology}

In the RRU system, there are M sectors. In the design of each sector, the link of the optical carrier antenna meets the RF design requirements, compared with the traditional design method. In the IFOF technology, the wavelength data information is required to be mastered in the network. In the design, the processing of the wavelength, there are certain shortcomings in the application of such technology, the capacity must be determined in advance. Relative to the design requirements of the enhanced signal, in the modulation management, non-linear analysis is performed. The signal of the long-term evolution enhanced system (LTE-A) is higher than the peak-to-average power ratio, so it is easier than the single-carrier modulated signal. Affected by nonlinear distortion.

\subsection{Physical layer function reconstruction fronthaul network}

In order to reduce the cost of running the fronthaul network, the optical band of the optical transmission needs to be controlled within the range of 10GB / S. IQ data compression technology can reduce the transmission bandwidth. [8]Currently, compressing one-half or one-third of the bandwidth cannot meet the transmission requirements. To solve the problem of transmission bandwidth, the most commonly used method is to transform the functional division point between RRH and BBU. This method mainly balances analog and digital processing schemes, and establishes a new structure based on split physical layer processing, which effectively reduces the bandwidth of optical transmission and also has the advantages of multi-point cooperative transmission.

\subsection{Pure Digital Signal General Public Radio Interface Protocol Fronthaul Network}

From the perspective of time efficiency, fronthaul network technology is still a common public wireless interface based on the existing 2.5Gbit / s and 5 Gbit / s. Its main advantage is that the overall layout is relatively simple and the cost is not high, which is especially suitable for setting.[9] It has high stability and reliable performance outdoors. This system mainly realizes the transmission of information through a link, and there is also a link that can monitor the transmission process of the link. The modified scheme can effectively reduce the demand for optical fiber. However, this technology can increase the burden of network management operations and maintenance. 


\section{Conclusion}

With the advent of the era of big data, the scale of network data continues to increase, which affects and limits network speed to a certain extent, network technology has undergone tremendous changes, and the theory of mobile communication network technology has continued to develop. As a core part of 5G, There is more general concern in the communications industry. At this stage, more researchers need to continue to explore the advantages of the key technologies of 5G communications fronthaul.

\section{Acknowledgment}

Topic name: Research and development of integrated receptacle with isolator for 5G fronthaul optical module NO: GJJ191224.

\section{References}

[1]Zhang J , Ji Y, Jia S , et al. Reconfigurable Optical Mobile Fronthaul Networks for Coordinated Multipoint Transmission and Reception in 5G[J]. Journal of Optical Communications and Networking, 2017, 9(6):489.

[2]Libo C , Ronghuan X , Zhongkai Z . Discussion on UTN Transmission Network Development for 5G Evolution[J]. Designing Techniques of Posts and Telecommunications, 2018.

[3]Kim B G , Bae S , Kim H , et al. RoF-based Mobile Fronthaul Networks Implemented by Using DML and EML for 5G Wireless Communication Systems[J]. Journal of Lightwave Technology, 2018:1-1.

[4]González, Sergio, Antonio D L O , Costa-Pérez, Xavier, et al. 5G-Crosshaul: An SDN/NFV control and data plane architecture for the 5G integrated Fronthaul/Backhaul[J]. Transactions on Emerging Telecommunications Technologies, 2016.

[5]Song S, Yejun L , Lei G, et al. Optimized relaying and scheduling in cooperative Free Space Optical fronthaul/backhaul of 5G[J]. Optical Switching and Networking, 2018, 30:62-70.

[6]Zhang J , Ji Y , Yu H , et al. Experimental demonstration of fronthaul flexibility for enhanced CoMP service in 5G radio and optical access networks[J]. Optics Express, 2017, 25(18):21247.

[7]Cvijetic N, Tanaka A , Kanonakis K , et al. SDN-controlled topology-reconfigurable optical mobile fronthaul architecture for bidirectional CoMP and low latency inter-cell D2D in the 5G mobile era[J]. Optics Express, 2014, 22(17):20809.

[8]Gohar M , Choi J G , Koh S J . TRILL-Based Mobile Packet Core Network for 5G Mobile Communication Systems[J]. Wireless Personal Communications, 2015, 87(1).

[9]Xia F , Xia Z Z , Huang X B . Research on Electric Power Communication Optical Fiber Transmission Network Evaluation and Optimization Method[J]. Advanced Materials Research, 2014, 1044-1045:1459-1462. 